\title{
Prospective study on the recurrence/metastasis of stage II/III colorectal cancer and gastric cancer associated with occult neoplastic cells in lymph node sinuses: Three-year interim results
}

\author{
MASAYA MUKAI ${ }^{1}$, TAKAYUKI TAJIMA ${ }^{1}$, SHINKICHI SATO ${ }^{2}$, HIROMI NINOMIYA ${ }^{2}$, \\ KANAKO WAKUI ${ }^{2}$, NOBUKAZU KOMATSU ${ }^{2}$, KAZUTOSHI TSUCHIYA ${ }^{3}$, \\ HISAO NAKASAKI $^{4}$ and HIROYASU MAKUUCHI ${ }^{5}$ \\ ${ }^{1}$ Department of Surgery, Tokai University Hachioji Hospital, Ishikawa-cho 1838, Hachioji, \\ Tokyo 192-0032; Departments of ${ }^{2}$ Pathology, ${ }^{3}$ Endoscopy Center, ${ }^{4}$ Surgery, Tokai University \\ Oiso Hospital, Gakyou 21-1, Oiso, Kanagawa 259-0198; ${ }^{5}$ Department of Surgery, \\ Tokai University School of Medicine, Bohseidai, Isehara, Kanagawa 259-1193, Japan
}

Received April 19, 2006; Accepted May 25, 2006

\begin{abstract}
This study was designed to prospectively examine whether the presence of occult neoplastic cells (ONCs) in lymph nodes or positive high-risk (HR) criteria were related to the survival of patients with stage II/III colorectal cancer or gastric cancer. The 3-year relapse-free survival (3Y-RFS) rate was calculated for 79 patients who were registered during a 2 -year period. The $3 \mathrm{Y}$-RFS rate was $80.5 \%$ in patients without ONCs $(n=54)$ and $84.3 \%$ in patients with ONCs $(\mathrm{n}=25 ; \mathrm{p}=0.9089)$. Among patients who had stage II/III colorectal cancer, it was $89.0 \%(n=47)$ and $76.2 \%(n=15)$, respectively $(\mathrm{p}=0.4131)$. For patients with stage III colorectal cancer alone, it was $80.8 \%(n=24)$ and $62.5 \%(n=9)$, respectively $(\mathrm{p}=0.4006)$. The $3 \mathrm{Y}-\mathrm{RFS}$ rate was respectively $88.1 \%$ and $77.6 \%$ for the HR patients $(n=31)$ and low-risk (LR) patients $(\mathrm{n}=48)$ with stage II/III colorectal cancer or gastric cancer $(\mathrm{p}=0.5545)$. It was respectively $92.3 \%$ and $84.3 \%$ for the HR patients $(n=20)$ and LR patients $(n=42)$ with stage II/III colorectal cancer $(\mathrm{p}=0.5073)$. Also, the rate
\end{abstract}

Correspondence to: Dr Masaya Mukai, Department of Surgery, Tokai University Hachioji Hospital, Ishikawa-cho 1838, Hachioji, Tokyo 192-0032, Japan

E-mail: mukai.masaya@hachioji-hosp.tokai.ac.jp

Abbreviations: ONCs, occult neoplastic cells; LNs, lymph nodes; 5-FU, 5-fluorouracil; LV, leucovorin; CPT-11, irinotecan; L-OHP, oxaliplatin; CDDP, cisplatin; TS, thymidylate synthase; DPD, dihydropyrimidine dehydrogenase; HR, high-risk; LR, low-risk

Key words: colorectal cancer, gastric cancer, occult neoplastic cells, micro-metastasis, isolated tumor cells, high-risk group, recurrence, metastasis was respectively $80 \%$ and $76.2 \%$ for the HR patients $(n=7)$ and LR patients $(n=26)$ with stage III colorectal cancer alone $(p=0.9506)$. These results indicate that the $3 Y-R F S$ rate is lower in ONC-positive patients with stage II/III colorectal cancer, suggesting that ONCs may have an influence on survival.

\section{Introduction}

Until the 1990s, the leading cause of cancer death among Japanese men was gastric cancer. With recent westernization of the diet and lifestyle, lung cancer has since replaced gastric cancer as the chief killer, followed by liver and colorectal cancer (1). In contrast, the leading cause of cancer death among Japanese women is still gastric cancer, followed by colorectal, lung, and breast cancer (1). Lymph node metastasis is known to be the most important clinical factor with an influence on survival in patients with malignancies of the digestive tract, such as gastric and colorectal cancer, or thoracic malignancies such as lung and breast cancer (2-5). It is thought that fatal recurrence/metastasis of such cancers occurs in the liver or lungs after surgical resection when free tumor cells or cell clusters circulate through the body during the perioperative period and escape the host immune system to survive and proliferate in these organs (6-9). Many studies have suggested that dissemination of viable cancer cells may be related to tumor recurrence/metastasis, and such tumor cells can be detected as cytokeratin-positive occult neoplastic cells (ONCs) floating in lymph node sinuses distant from the primary tumor (10-18). These ONCs can be counted after immunostaining and represent a few malignant cells trapped by the lymph nodes (LNs) (7-10). Free cancer cells are mentioned as isolated tumor cells in the sixth version of the TNM classification. However, these cells are only defined as cells with a diameter of $<0.2 \mathrm{~mm}$ that can be detected by routine $H \& E$ staining, so the number of such cancer cells that 
represents a significant risk and the handling of tumor cell clusters remain undecided (19). In contrast, ONCs have been clearly defined as cells floating freely in LN sinuses distant from the primary tumor that can only be detected by cytokeratin immunostaining, and include cell clusters $(<10$ cells) as well as malignant micro-aggregates ( $\geq 10$ cells) (20). It has been reported that ONCs may induce recurrence/ metastasis in any organ, and that these cells should be clearly distinguished from isolated tumor cells or micro-metastases anchored in LNs, and should be classified as more malignant occult systemic metastases (20).

ONCs were reported to have a sensitivity, specificity, positive predictive value (PPV), and negative predictive value (NPV) of 77, 100, 100, and 71\%, respectively, for predicting the recurrence of Dukes' B primary colorectal cancer, while the respective values were $75,72,73$, and $74 \%$ for Dukes' C cancer (10). Investigation of ONCs seems particularly useful in patients with Dukes' B cancer, since negativity for ONCs may be a very reliable indicator of a low risk of recurrence in Dukes' B patients (10). To increase the sensitivity and PPV, for detecting high-risk patients have been developed to predict recurrence by combining ONCs with clinicopathological features, and these criteria have been reported to be useful $(9,15-17)$. It has been reported that patients who fulfill any two of the following three criteria have a high risk of suffering from recurrence/metastasis of colorectal/gastric cancer without LN metastasis: 1) lymphatic invasion (ly) and venous invasion (v) are positive in the primary tumor; 2) fewer than 14 LNs are dissected at surgery; and 3) ONCs are positive in LN sinuses $(9,11,14,15,17,18)$. It has also been reported that patients who have colorectal or gastric cancer with LN metastasis and satisfy any two of the following three criteria have a high risk of developing recurrence/metastasis: 1) patients in whom D2,3 (dissection number; extent of LN dissection) is not greater than $\mathrm{n} 1$ (n-number; extent of LN metastasis) and who have $\geq 3$ metastatic nodes; 2) patients in whom the depth of tumor invasion is se (serosal exposure)/a2 (adventitial exposure); and 3) patients who are positive for ONCs $(9,12,13,16,18)$.

However, a prospective clinical trial has not yet been performed to assess the value of these criteria for detecting patients with a high risk of recurrence/metastasis. Accordingly, this study was designed to prospectively examine whether detection of ONCs in LN sinuses or the high-risk criteria were related to the $3 \mathrm{Y}-\mathrm{RFS}$ rate of patients with stage II/III primary colorectal cancer or gastric cancer.

\section{Patients and methods}

After obtaining the approval of the Medical Ethics Committee of Tokai University School of Medicine, patients who met the following criteria were enrolled as eligible from among 104 patients with stage II/III primary colorectal cancer or gastric cancer who underwent curative resection from October 2002 to October 2004: 1) patients with an age $<75$ years and performance status of 0 or 1 (Japan Cancer Therapy Association); 2) patients with accurate and complete medical records who were traceable for assessment of recurrence; and 3) patients from whom at least ten histologically negative LNs showing no metastasis on routine H\&E staining were
Table I. Criteria for separating patients into groups with a high risk or low risk of the recurrence/metastasis of nodenegative $(\mathrm{LN}-)$ or node-positive $(\mathrm{LN}+)$ colorectal cancer and gastric cancer.

\begin{tabular}{lcc}
\hline & \multicolumn{2}{c}{ Recurrence and metastasis } \\
\cline { 2 - 3 }$\geq 2$-factors & Low-risk group & High-risk group \\
\hline LN (-) & Others & ly $(+)$ and $\mathrm{v}(+)$ \\
& LN dissection $\geq 15$ nodes & $\leq 14$ nodes \\
& Occult neoplastic cells $(-)$ & $(+)$ \\
LN (+) & D2,3 $>$ nl $(<3$ nodes $)$ & Others \\
& Tumor invasion $\leq$ ss/al & $\geq$ se/a2 \\
& Occult neoplastic cells $(-)$ & $(+)$ \\
\hline
\end{tabular}

LN, lymph node; ss, subserosal invasion; se, serosal exposure; al, subadventitial invasion; a2, adventitial exposure.

collected at the time of surgery. A total of 79 patients were registered as eligible, including 62 patients with colorectal cancer (29 in stage II and 33 in stage III) and 17 patients with gastric cancer ( 5 in stage II and 12 in stage III). The patients were examined for ONCs and the high-risk criteria within three weeks after surgery to divide them into ONC-negative $(-)$ and $\mathrm{ONC}$-positive (+) groups as well as into high-risk and low-risk groups. Then data on tumor recurrence and metastasis were collected.

Postoperative chemotherapy. Based on the standard first-line adjuvant chemotherapy used in Japan, $400 \mathrm{mg} / \mathrm{m}^{2}$ of UFT (Tegafur/Uracil, Taiho Pharmaceutical Co., Ltd., Tokyo, Japan) and $3.0 \mathrm{~g}$ of the biological response modifier PSK (Krestin; Kureha Chemical Industry Co., Ltd., Tokyo, Japan) were administered orally for three years to the patients with stage II colorectal cancer $(21,22)$. Patients with stage III colorectal cancer were treated with $350 \mathrm{mg} / \mathrm{m}^{2}$ of 5 -fluorouracil (5-FU) and $150 \mathrm{mg} / \mathrm{m}^{2}$ of leucovorin (LV) 5 times weekly by intravenous infusion for six months, followed by oral administration of UFT plus PSK for 2.5 years $(21,22)$. Patients who had stage II/III gastric cancer were treated with $350 \mathrm{mg} / \mathrm{m}^{2}$ of 5-FU and low-dose cisplatin (CDDP; $5 \mathrm{mg} / \mathrm{m}^{2}$ ) 5 times weekly by intravenous infusion for six months, followed by oral administration of UFT plus PSK for 2.5 years $(23,24)$.

The patients were asked to complete a questionnaire to evaluate their postoperative QOL and drug compliance at one year after surgery. A doctor, nurse, or pharmacist collected and checked the questionnaire. The day when recurrence was confirmed by CT and/or US was defined as the time of recurrence. All of the patients were assessed for survival during the one-month period from December 2005 to January 2006 , and the data thus obtained were used to calculate the 3 Y-RFS rate.

Detection of ONCs in lymph node sinuses. Immunohistochemical detection of cytokeratin in harvested LN was performed by the indirect immunoperoxidase method using a monoclonal anti-cytokeratin antibody (AE1/AE3; Fuji Chemical Industries, Ltd., Japan) $(25,26)$. A total of 10 LNs 


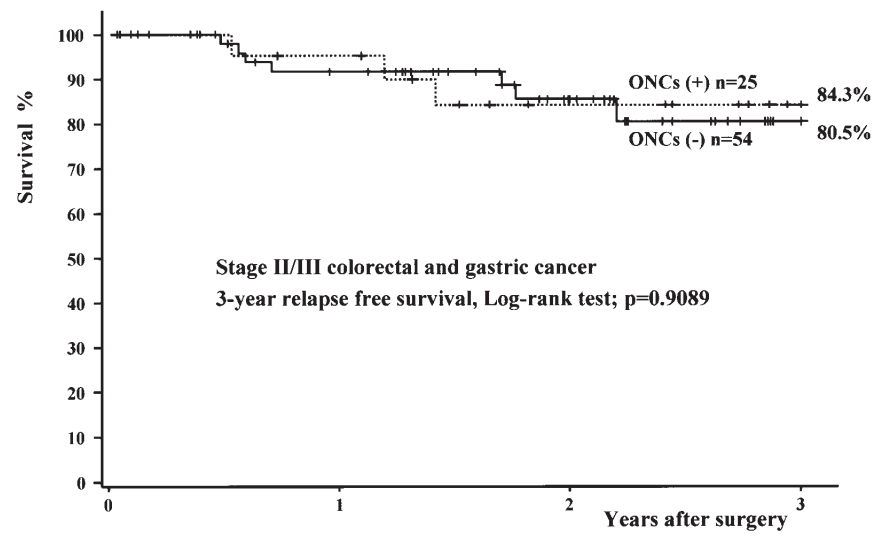

Figure 1. Among patients with stage II/III colorectal cancer and gastric cancer, the 3-year relapse-free survival rate was $80.5 \%$ when ONCs were negative $(n=54)$ and $84.3 \%$ when ONCs were positive $(n=25)$. There was no significant difference between the two groups $(\mathrm{p}=0.9089)$.

that were negative for metastasis by routine H\&E staining were randomly selected from each patient. Accordingly, a total of 790 nodes were selected from 79 patients. Serial sections of each LN were stained with H\&E and keratinAE1/AE3, after which the positive rate and positive cell count was determined for each stain. Tumor cells and/or tumor cell nests anchored in the LNs were excluded when counting ONCs and only cells floating freely in the LN sinuses were included. Immunostaining for cytokeratin was assessed as positive when $\geq 3$ cytokeratin-positive cells were detected, while it was defined as negative for 0-2 cytokeratin-positive cells (6-9).

High-risk (HR) group and low-risk (LR) group for recurrencel metastasis of colorectal and gastric cancer

Stage II colorectal cancer and node-negative stage II/III gastric cancer. Lymph node-negative patients who met at least two of the following three criteria were classified into the high-risk (HR) group for recurrence/metastasis: i) the primary tumor was positive for both lymphatic invasion (ly) and venous invasion (v); ii) the number of LNs harvested during surgery was $\leq 14$; and iii) ONCs were positive in LN sinuses by cytokeratin immunostaining $(9,11,14,15,17,18)$ (Table I).

Stage III colorectal cancer and node-positive stage II/III gastric cancer. Lymph node-positive patients who met at least two of the following three criteria were classified into the HR group for recurrence/metastasis: i) those who were not $\mathrm{D} 2,3>\mathrm{n} 1$ and did not have $<3$ histologically positive metastatic LNs; ii) those in whom histological tumor invasion had progressed beyond the subserosal/subadventitial layer ( $\leq$ ss/a1) to serosal/adventitial exposure ( $\geq$ se/a2); and iii) those positive for ONCs in the LN sinuses by cytokeratin immunostaining $(9,12,13,16,18)$ (Table I).

Statistical analysis. The 3-year RFS rate was calculated by the Kaplan-Meier method and the log-rank test was used for comparison between the ONC (+) or ONC (-) groups and the HR or LR groups. A p-value of $<0.05$ was considered to

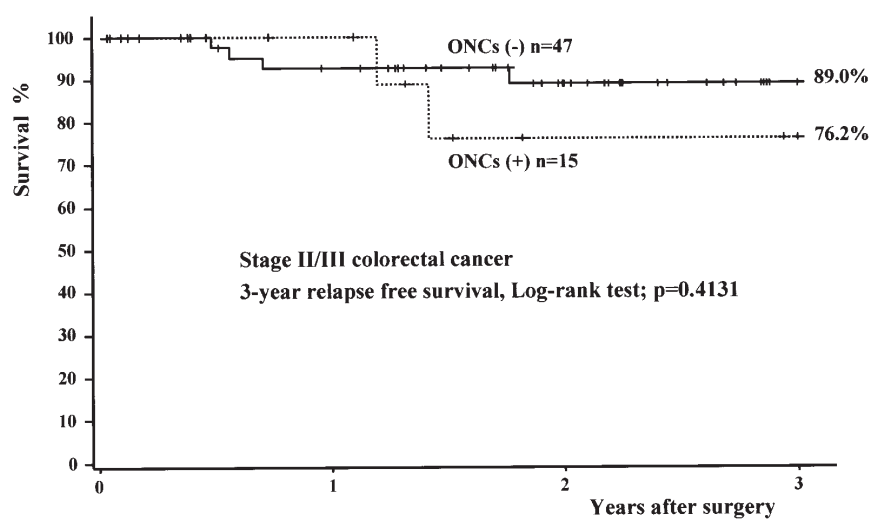

Figure 2. Among patients with stage II/III colorectal cancer, the 3-year relapse-free survival rate was $89.0 \%$ when ONCs were negative $(n=47)$ and $76.2 \%$ when ONCs were positive $(n=15)$. There was no significant difference between the two groups $(\mathrm{p}=0.4131)$.

indicate a significant difference. All analyses were done with SPSS 13.0 J software (SPSS Japan, Inc., Tokyo, Japan).

\section{Results}

Tolerability and compliance with adjuvant chemotherapy. Among 33 patients with stage III colorectal cancer, 29 patients $(87.9 \%)$ completed at least five courses of adjuvant chemotherapy, three patients completed four courses, and one patient completed two courses. However, administration was changed from the intravenous to oral route in all of these patients. Compliance was achieved for at least six months in $93.9 \%$ (31/33) of patients with stage III colorectal cancer and in $96.6 \%$ $(28 / 29)$ of patients with stage II colorectal cancer. Among 17 patients with stage II/III gastric cancer, 14 patients $(82.4 \%)$ completed at least five courses of adjuvant chemotherapy, two patients completed three courses, and one patient completed two courses. Administration was switched from the intravenous to oral route in all of the patients. Compliance was achieved for at least six months in $88.2 \%(15 / 17)$ of the patients with stage II/III gastric cancer. None of the patients experienced grade 4 adverse events during the administration period.

ONCs in lymph node sinuses. Among patients with stage II/III colorectal or gastric cancer, the $3 \mathrm{Y}$-RFS rate was $80.5 \%$ when ONCs were negative $(n=54)$ and $84.3 \%$ when ONCs were positive $(n=25)(p=0.9089$; Fig. 1). Among patients with stage II/III colorectal cancer, the $3 \mathrm{Y}$-RFS rate was respectively $89.0 \%(n=47)$ and $76.2 \%(n=15)(p=0.4131$; Fig. 2$)$. For patients with stage III colorectal cancer alone, the 3Y-RFS rate was $80.8 \%(n=24)$ and $62.5 \%(n=9)$, respectively ( $\mathrm{p}=0.4006$; Fig. 3). For patients with stage II/III gastric cancer, the rate was $57.1 \%(\mathrm{n}=7)$ and $90.0 \%(\mathrm{n}=10)$, respectively $(\mathrm{p}=0.0830)$, while it was respectively $75.0 \%(\mathrm{n}=4)$ and $87.5 \%$ $(\mathrm{n}=8)$ for patients with stage III gastric cancer alone $(\mathrm{p}=0.6528)$.

High-risk (HR) and low-risk (LR) groups. Among patients with stage II/III colorectal or gastric cancer, the 3 Y-RFS rate was $88.1 \%$ in the HR group $(n=31)$ and $77.6 \%$ in the LR group $(\mathrm{n}=48)(\mathrm{p}=0.5545$; Fig. 4$)$. The rate was respectively 92.3 and 


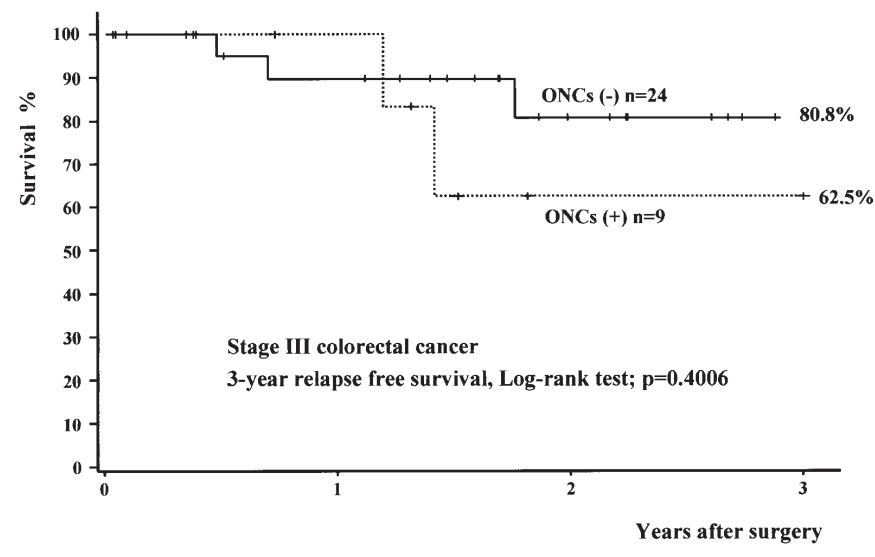

Figure 3. Among patients with stage III colorectal cancer alone, the 3-year relapse-free survival rate was $80.8 \%$ when ONCs were negative $(\mathrm{n}=24)$ and $62.5 \%$ when ONCs were positive $(n=9)$. There was no significant difference between the two groups $(\mathrm{p}=0.4006)$.

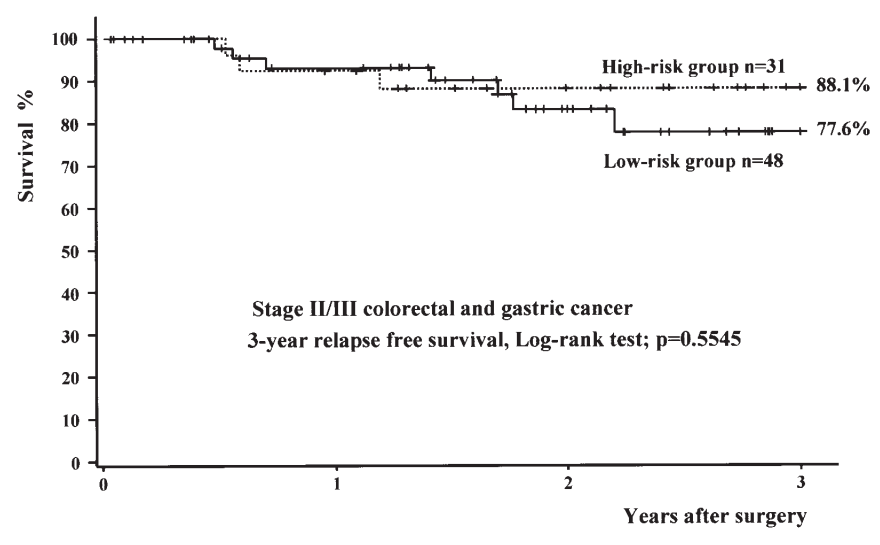

Figure 4. Among patients with stage II/III colorectal cancer or gastric cancer, the 3-year relapse-free survival rate was $88.1 \%$ for the high-risk group $(n=31)$ and $77.6 \%$ for the low-risk group $(n=48)$. There was no significant difference between the two groups $(\mathrm{p}=0.5545)$.

$84.3 \%$ in the HR group $(n=20)$ and LR group $(n=42)$ of patients with stage II/III colorectal cancer ( $\mathrm{p}=0.5073$; Fig. 5). Also, the rate was respectively 80 and $76.2 \%$ in the HR group $(n=7)$ and LR group $(n=26)$ of patients with stage III colorectal cancer alone ( $\mathrm{p}=0.9506$; Fig. 6). The 3 Y-RFS rate was respectively 78.6 and $66.7 \%$ in the HR group $(n=14)$ and LR group $(n=3)$ of patients with stage II/III gastric cancer $(\mathrm{p}=0.5063)$, while it was respectively 81.8 and $100 \%$ in the HR group $(n=11)$ and LR group $(n=1)$ of patients with stage III gastric cancer alone $(\mathrm{p}=0.6622)$.

\section{Discussion}

Criteria for classifying breast cancer into local or systemic disease based on the presence/absence of $\mathrm{LN}$ metastasis have long been established. The criteria for selecting patients with a high risk of suffering from the recurrence/metastasis of breast cancer have also been examined in detail, and the therapeutic regimens and anticancer drugs for $H R$ and LR cases have been determined $(3,27,28)$. With respect to colorectal cancer, various multicenter randomized studies have been reported

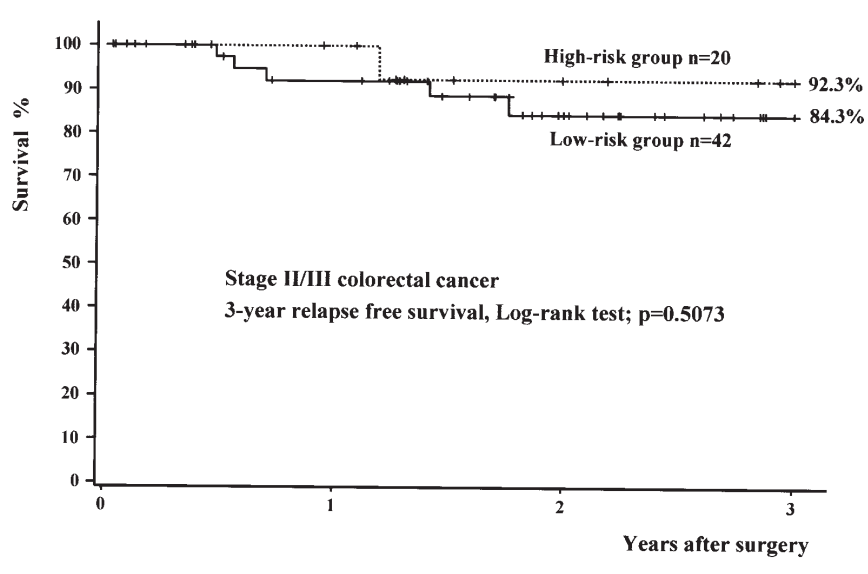

Figure 5. Among patients with stage II/III colorectal cancer, the 3-year relapse-free survival rate was $92.3 \%$ for the high-risk group $(n=20)$ and $84.3 \%$ for the low-risk group $(n=42)$. There was no significant difference between the two groups $(\mathrm{p}=0.5073)$.

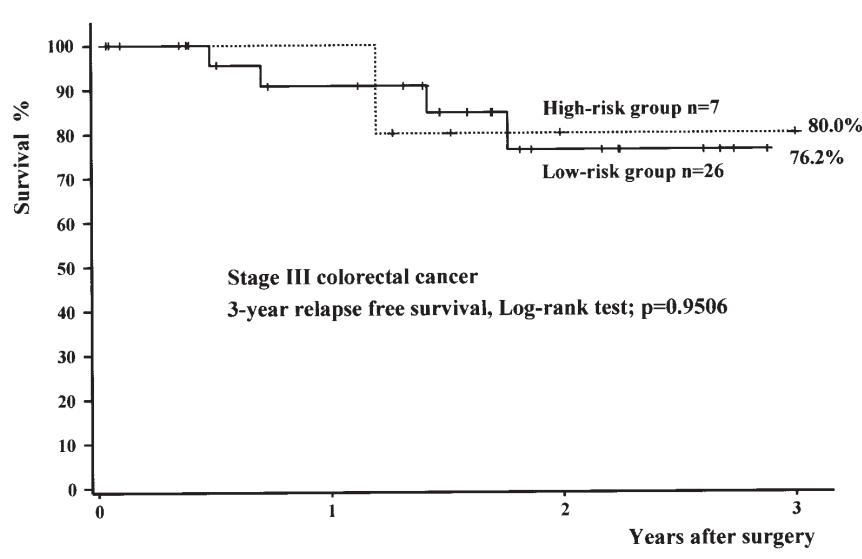

Figure 6. Among patients with stage III colorectal cancer alone, the 3-year relapse-free survival rate was $80.0 \%$ for the high-risk group $(n=7)$ and $76.2 \%$ for the low-risk group $(n=26)$. There was no significant difference between the two groups $(\mathrm{p}=0.9506)$.

in western countries, such as NSABP-C06 (Wolmark N, et al, Proc ASCO 40: abs. 3508, 2004), and multicenter randomized studies in patients with gastric and colorectal cancer has just been started in Japan, but no clear selection criteria for HR patients of cancer of the digestive tract have been specified. Since prospective studies usually divide subjects into HR and LR groups and then assign appropriate therapies for the two groups, it is necessary to register a large number of subjects and a single institution or small group cannot handle such large-scale studies. In the present study, patients with each stage of cancer and received with stage-specific anticancer therapy, thus eliminating the need to consider the possibility of differences in the rate of recurrence/metastasis due to different therapeutic regimens. Interim analysis has not been performed for stage II/Dukes' B colorectal cancer because only one such patient has experienced recurrence so far. Final analysis will be completed in November 2009.

Unlike micrometastases and isolated tumor cells, for which a relationship to survival remains controversial due to the lack of a clear definition, it has been consistently reported that 
occult systemic metastases represented by ONCs are associated with an increased risk of recurrence/metastasis (20,29-32). To make postoperative adjuvant chemotherapy more effective for suppressing recurrence/metastasis after curative resection of the primary tumor, it is necessary to perioperatively eliminate residual cancer cells that have the potential to spread throughout the body. For this purpose, systemic consolidation chemotherapy delivered soon after surgery should be most effective. If the ONCs (+) group or HR group in patients with stage II/III cancer has a significantly worse prognosis, it can be assumed that adjuvant chemotherapy failed to eliminate ONCs, particularly clusters of these cells (20). Earlier studies have investigated the percentage of patients with circulating ONC clusters sensitive to 5-FU/LV therapy at the time of spread to distant organs, and it has been shown that there were only a few sensitive tumor cells in patients with high-TS activity and low-DPD activity, suggesting that the potency of chemotherapy may have been inadequate (29-32). Multi-drug combination therapy using CPT-11 and/or L-OHP, which is more popular in western countries than Japan, may be more effective for patients with tumors that are resistant to 5-FU/ LV therapy $(29,30)$. Although the administration method and the doses used have an influence, the sensitivity of a tumor to the combination of anticancer drugs given is the most important factor determining the response. In the present study, 5-FU/LV was used as the basal therapy for patients who had stage III cancer with ONCs. From the viewpoint of drug sensitivity for ONC-clusters, the addition of CPT-11 and/or L-OHP may have increased the effect, but detailed investigation of the best drugs for combination therapy is still necessary (29-32).

It has been reported that the incidence of postoperative peritoneal dissemination is about $10 \%$ for colorectal cancer, which is much lower than for gastric cancer (33). Although long-term survival of stage IV colorectal cancer patients without peritoneal dissemination has been reported, the prognosis is very poor after occurrence of peritoneal dissemination (33). Colorectal cancer differs from gastric cancer with respect to the mode of recurrence/metastasis and its biological behavior. Even when colorectal cancer metastasizes and to distant organs such as the liver and lungs, it can be treated by debulking surgery with a relatively good outcome as long as the metastasis is a solitary nodule or there is a small number of resectable lesions $(34,35)$. Stage II/III gastric cancer positive for LN metastasis is associated with peritoneal dissemination in at least $30-40 \%$ of patients who undergo surgery. There are two known mechanism of peritoneal dissemination, which are tumor cell dissemination to the peritoneal lining via lymphatics and direct spread of free cancer cells from sites of serosal exposure followed by survival/proliferation in the peritoneal cavity (33). It is also possible that gastric cancer may metastasize to intramural LNs, enter the greater omentum via lymphatics, and then be directly implanted into the abdominal wall and peritoneum (33). In the present study, four out of seven patients $(57.1 \%)$ with ONC-negative stage II/III gastric cancer experienced recurrence, and three of these four patients had advanced recurrence with peritoneal dissemination. Thus, ONCs may serve as a useful clinical indicator of the risk of hematogenous recurrence/metastasis of colorectal cancer to the liver and lungs, but not as an indicator of recurrence/metastasis for patients with gastric cancer, particularly in the case of peritoneal dissemination. Because it was reported that ONCs are detected in stage Ic, but not stage Ia, ovarian cancer positive for tumor cells on perioperative peritoneal cytology (36), it can be inferred that gastric cancer metastasizes by releasing tumor cells directly into the peritoneal cavity from sites of serosal exposure rather than via lymph flow. It is also possible that there may be organ-specific forms of tumor dissemination.

It has been reported that improving the survival rate after recurrence is the most important factor for increasing the overall survival of cancer patients $(37,38)$. However, it is quite difficult to determine the effect of treatment after recurrence because of the wide variety of therapeutic options that are used, including surgical resection with or without chemotherapy and/or chemo-radiation therapy, as well as hepatic artery infusion therapy. In general, the effect of chemoradiation therapy and/or chemotherapy in unresected patients is evaluated from indexes such as the response rate, progression-free survival, and time to progression, although such results do not include patients who undergo additional surgical resection, which is known to produce a relatively good outcome. When the effect of multidisciplinary therapy for recurrence has been evaluated based on the 5-year overall survival rate, the results have been very poor $(1,4,6,33)$. It is important to treat patients who have ONC-positive stage II/III colorectal cancer with efficient adjuvant therapy that targets circulating cells soon after radical resection and before overt recurrence, thereby eliminating occult metastatic foci in the early postoperative period. Such treatment should achieve a marked improvement of the 5-year relapse-free survival rate. In conclusion, our interim analysis showed that patients with stage II/III colorectal cancer who were positive for ONCs had a lower 3 Y-RFS rate, suggesting that ONCs may influence survival.

\section{Acknowledgements}

This study was supported by grants from the Occult Neoplastic Cells Research and Study Group (No. 2005-2291; Tokai University Hachioji Hospital, Hachioji, Tokyo, Japan) and the Research and Study Program of Tokai University Educational System General Research Organization (No. 2005-31; Tokai University Hospital, Isehara, Kanagawa, Japan).

\section{References}

1. Journal of Health and Welfare Statistics, Health and Welfare Statistics Association, Tokyo, 51: 46-49, 2004.

2. Omejc M, Juvan R, Jelenc F and Repse S: Lymph node metastases in gastric cancer: correlation between new and old UICC TNM classification. Int Surg 86: 14-19, 2001.

3. Goldhirsch A, Glick JH, Gelber RD, Coates AS and Senn H-J: Meeting highlights: International consensus panel on the treatment of primary breast cancer. J Clin Oncol 19: 3817-3827, 2001.

4. Multi-institutional registry of large bowel cancer in Japan, cases treated in 1994. In: Japanese Society for Cancer of the Colon and Rectum. Vol. 23, Tokyo, 2002.

5. Prenzel KL, Mönig SP, Sinning JM, Baldus SE, Brochhagen H-G, Schneider PM and Hölscher AH: Lymph node size and metastatic infiltration in non-small cell lung cancer. Chest 123: 463-467, 2003. 
6. Mukai M, Ito I, Mukoyama S, Tajima T, Saito Y, Nakasaki H, Sato $\mathrm{S}$ and Makuuchi H: Improvement of 10 -year survival by Japanese radical lymph node dissection in patients with Dukes' B and C colorectal cancer: a 17-year retrospective study. Oncol Rep 10: 927-934, 2003.

7. Mukai M, Sato S, Komatsu N, Nishida T, Shiba K, Ito I, Nakasaki $\mathrm{H}$ and Makuuchi H: Correlation between occult neoplastic cells in the lymph node sinuses and recurrence in patients with Dukes' C colorectal cancer. Oncol Rep 10: 1165$1169,2003$.

8. Mukai M, Sato S, Komatsu N, Nishida T, Shiba K, Ito I, Nakasaki H and Makuuchi H: Correlation between occult neoplastic cells in the lymph node sinuses and recurrence in patients with curatively resected Dukes' B colorectal cancer. Oncol Rep 10: 1177-1181, 2003.

9. Mukai M, Sato S, Nishida T, Komatsu N, Shiba K, Nakasaki H and Makuuchi H: Selection criteria for high-risk and low-risk groups of recurrence and metastasis in patients with primary colorectal cancer. Oncol Rep 10: 1753-1758, 2003.

10. Mukai M, Sato S, Nakasaki H, Saito Y, Nishiumi N, Iwasaki M, Tokuda Y, Ogoshi K, Inoue H, and Makuuchi H: Occult neoplastic cells in the lymph node sinuses and recurrence of primary breast, lung, esophageal and gastric cancer. Oncol Rep 11: 81-84, 2004.

11. Mukai M, Sato S, Komatsu N, Kimura T, Ninomiya H, Nakasaki H, Ogoshi K and Makuuchi M: Accuracy of criteria for predicting the recurrence and metastasis of stage I and II gastric cancer without lymph node metastasis. Oncol Rep 12: 59-62, 2004.

12. Mukai M, Sato S, Komatsu N, Kimura T, Ninomiya H, Nakasaki H, Ogoshi K and Makuuchi H: Accuracy of criteria for predicting recurrence and metastasis in stage II and III gastric cancer with lymph node metastasis. Oncol Rep 12: 6366, 2004

13. Mukai M, Sato S, Komatsu N, Kimura T, Ninomiya H, Nakasaki H, Ogoshi K and Makuuchi H: Predicting the recurrence/metastasis of stage II and III breast cancer with lymph node metastasis. Oncol Rep 12: 303-306, 2004.

14. Mukai M, Sato S, Komatsu N, Kimura T, Ninomiya H, Nakasaki H, Ogoshi K and Makuuchi H: Predicting the recurrence/metastasis of stage I and II breast cancer without lymph node metastasis. Oncol Rep 12: 745-748, 2004.

15. Mukai M, Sato S, Tajima T, Kimura T, Komatsu N, Ninomiya H, Nakasaki H, Ogoshi K and Makuuchi H: Predicting recurrence and metastasis of stage II/Dukes' B colorectal cancer without lymph node metastasis. Oncol Rep 12: 1127-1130, 2004.

16. Mukai M, Sato S, Tajima T, Kimura T, Komatsu N, Ninomiya H, Nakasaki H, Ogoshi K and Makuuchi H: Predicting recurrence and metastasis of stage III/Dukes' C colorectal cancer with lymph node metastasis. Oncol Rep 12: 1301-1304, 2004.

17. Mukai M, Sato S, Tajima T, Kimura T, Komatsu N, Ninomiya H, Nakasaki H, Ogoshi K and Makuuchi H: Predicting recurrence and metastasis of Dukes' A primary colorectal cancer with or without proper muscle invasion. Oncol Rep 12: 1305-1308, 2004.

18. Tajima T, Mukai M, Sato S, Wakui K, Ninomiya H, Komatsu N, Nakasaki $\mathrm{H}$ and Makuuchi $\mathrm{H}$ : Predicting recurrence and metastasis of primary esophageal cancer with or without lymph node metastasis. Oncol Rep 15: 809-814, 2006.

19. TNM Classification of Malignant Tumours. 6th edition, John Wiley \& Sons, Inc., New York, NY, 2002.

20. Mukai M: Occult neoplastic cells and malignant microaggregates in lymph node sinuses: review and hypothesis. Oncol Rep 14: 173-175, 2005.

21. Mukai M, Tajima T, Nakasaki H, Sato S, Ogoshi K and Makuuchi H: Efficacy of postoperative adjuvant oral immunochemotherapy in patients with Dukes' B colorectal cancer. Ann Cancer Res Therap 11: 201-214, 2003.

22. Mukai M, Tajima T, Nakasaki H, Sato S, Ogoshi K and Makuuchi H: Efficacy of postoperative adjuvant oral immunochemotherapy in patients with Dukes' C colorectal cancer. Ann Cancer Res Therap 11: 215-229, 2003.
23. Enjoji A and the Nagasaki digestive organ cancer chemotherapy study group: Combination chemotherapy of 5-fluorouracil and low-dose Cisplatin in advanced and recurrent gastric cancer: a multicenter retrospective study in Nagasaki, Japan. Anticancer Res 22: 1135-1140, 2002

24. Mukai M, Hinoki T, Tajima T, Sato S, Nakasaki H, Ogoshi K and Makuuchi H: Complete remission after combination chemotherapy for stage IV gastric cancer with peritoneal dissemination and liver matastases; case report. Ann Cancer Res Therap 11: 169-181, 2003.

25. Nakane PK and Pierce GB: Enzyme-labeled antibodies: preparation and application for localization of antigens. $J$ Histochem Cytochem 14: 929-931, 1966.

26. Nakane PK and Pierce GB: Enzyme-labeled antibody for the light and electron microscopic localization of tissue antigens. J Cell Biol 33: 307-318, 1967.

27. National Institutes of Health Consensus Development Panel: National Institutes of Health consensus development conference statement: adjuvant therapy for breast cancer, November 1-3, 2000. J Natl Cancer Inst 93: 979-989, 2001.

28. Abrams JS: Adjuvant therapy for breast cancer-results from the USA consensus conference. Breast Cancer 8: 298-304, 2001

29. Mukai M, Sato S, Ninomiya H, Wakui K, Komatsu N, Tsuchiya K, Nakasaki H and Makuuchi H: Recurrence and 5-FU sensitivity of stage III/Dukes' C colorectal cancer with occult neoplastic cells in lymph node sinuses. Oncol Rep 14: 1165-1169, 2005.

30. Mukai M, Sato S, Ninomiya H, Wakui K, Komatsu N, Tsuchiya K, Nakasaki H and Makuuchi H: Recurrence and 5-FU sensitivity of stage II/Dukes' B colorectal cancer with occult neoplastic cells in lymph node sinuses. Oncol Rep 14: 1171-1176, 2005.

31. Mukai M, Sato S, Ninomiya H, Wakui K, Komatsu N, Tsuchiya K, Nakasaki H and Makuuchi H: Recurrence and 5-FU sensitivity of node-positive stage II/III gastric cancer with occult neoplastic cells in lymph node sinuses. Oncol Rep 14: 1505-1510, 2005.

32. Mukai M, Sato S, Ninomiya H, Wakui K, Komatsu N, Tsuchiya K, Nakasaki H and Makuuchi H: Recurrence and 5-FU sensitivity of stage I/II node-negative breast, lung, or gastric cancer with occult neoplastic cells in lymph node sinuses. Oncol Rep 15: 815-820, 2006.

33. Hasegawa S, Mukai M, Sato S, Tajima T, Wakui K, Ninomiya H, Komatsu N, Nakasaki N and Makuuchi H: Long-term survival and tumor 5-FU sensitivity in patients with stage IV colorectal cancer and peritoneal dissemination. Oncol Rep 15: 1185-1190, 2006.

34. Galandiuk S, Wieand HS, Moertel CG, Cha SS, Fitzgibbons RJ, Pemberton JH and Wolff BG: Pattern of recurrence after curative resection of carcinoma of the colon and rectum. Surg Gynecol Obst 174: 27-32, 1992.

35. Ambiru S, Miyazaki M, Ito H, Nakagawa K, Shimizu H, Kato A, Nakamura S, Omoto $\mathrm{H}$ and Nakajima N: Resection of hepatic and pulmonary metastases in patients with colorectal carcinoma. Cancer 82: 274-278, 1998.

36. Muramatsu T, Mukai M, Sato S, Tajima T, Nagase E, Ikeda M, Goya K, Shida M, Hirasawa T, Murakami and Shinozuka T: Clinical usefulness of serum and immunohistochemical markers in patients with stage Ia and Ic ovarian cancer. Oncol Rep 14: 861-865, 2005.

37. Feinstein AR, Sosin DM and Wells CK: The Will Rogers phenomenon: stage migration and new techniques as a source of misleading statistics for survival in cancer. N Engl J Med 312: $1604-1608,1985$.

38. Bunt AMG, Hermans J, Smit VTHBM, van de Velde CJH, Fleuren GL and Bruijn JA: Surgical/pathologic stage migration confounds comparisons of gastric cancer survival rates between Japan and Western countries. J Clin Oncol 13: 19-25, 1995. 